\title{
Successful drainage of recurrent pancreatic pseudocyst via a transpapillary and transpancreatic approach, using a conventional cystotome
}

A 54-year-old patient was previously admitted to our hospital in 2010 with a history of biliary necrotizing pancreatitis and pancreatic fluid collection with necrotic debris projecting into the tail of the pancreas. We carried out endoscopic ultrasound-guided (EUS) transgastric drainage of the superinfected necrotic area. The patient was discharged and 6 months later the pigtails were removed after complete resolution of the fluid collection. After another 6 months, transabdominal ultrasound showed a large pseudocyst at the same site without any signs of inflammation. The diagnosis was confirmed by computed tomography (CT) ( Fig. 1). EUS revealed a large stenosis in the pancreatic duct and there was suspicion of a fistula in relation to the pseudocyst. Transgastric access for pseudocyst drainage was impeded by the presence of multiple varices because of splenic vein thrombosis. We therefore chose a transpapillary approach for draining the pseudocyst (diameter $10 \mathrm{~cm}$ ).

Endoscopic retrograde pancreatography (ERP) confirmed the presence of filiform stenosis in the pancreatic main duct and a prestenotic communication into the pseudocyst ( Fig.2). While a guide wire passed through the stenosis, neither an ERCP cannula nor a biliary dilatation catheter could be passed across it. After taking informed consent, another attempt was made to place the guide wire again via the stenosis into the pancreatic pseudocyst ( Fig.3). A cystotome was placed in the stenosis and moved under blended current into the pseudocyst ( $\bullet$ Fig.4). A nasocystic tube was placed, which spontaneously drained $500 \mathrm{~mL}$ of clear cystic fluid ( Fig.5). A follow-up CT scan revealed rapid and complete resolution of the pseudocyst, and 4 days later the patient had was discharged with a $17-\mathrm{cm}$, 7-F Amsterdam stent. There were no signs of discomfort or inflammation. This case demonstrates that access to a pancreatic pseudocyst for transpapillary drainage through a stenosed duct can be gained with a cystotome under blended current, so long as the guide wire is safely placed inside the pseudocyst.

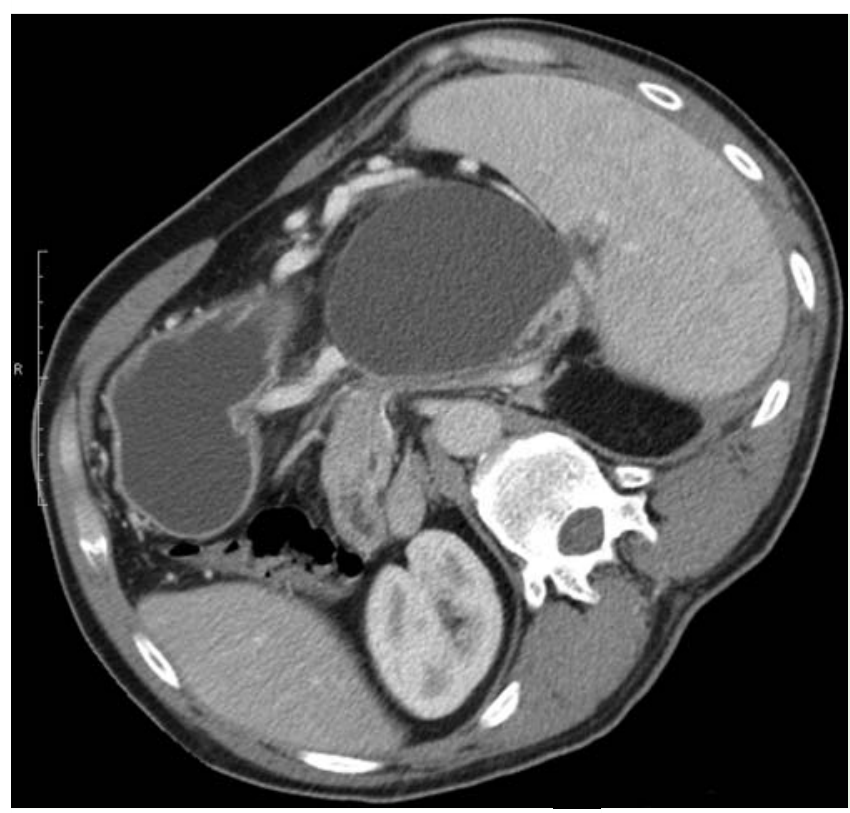

Fig. 1 Computed tomography (CT) scan showing pancreatic pseudocyst in a patient with history of biliary necrotizing pancreatitis and pancreatic fluid collection with necrotic debris projecting into the tail of the pancreas.

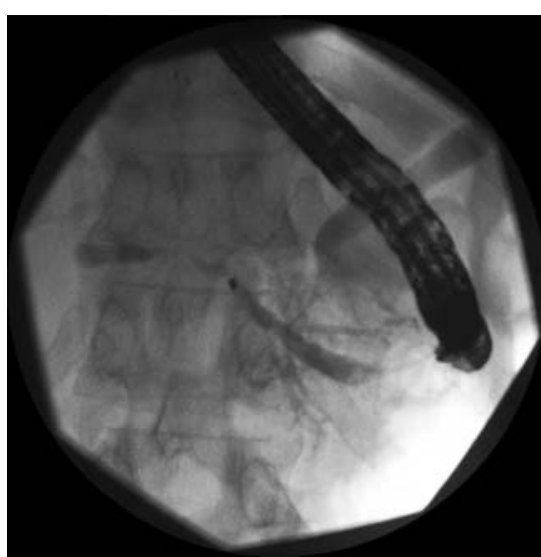

Fig. 2 Stenosis of the pancreatic duct and the pancreatic fistula.

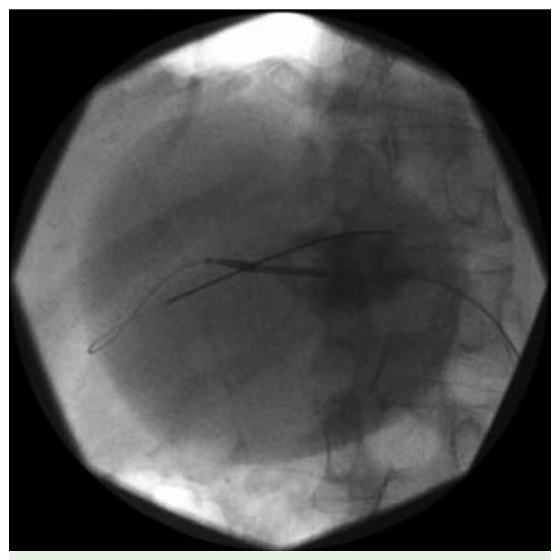

Fig. 4 Guide wire-assisted transpancreatic access to the pseudocyst using a cystotome.

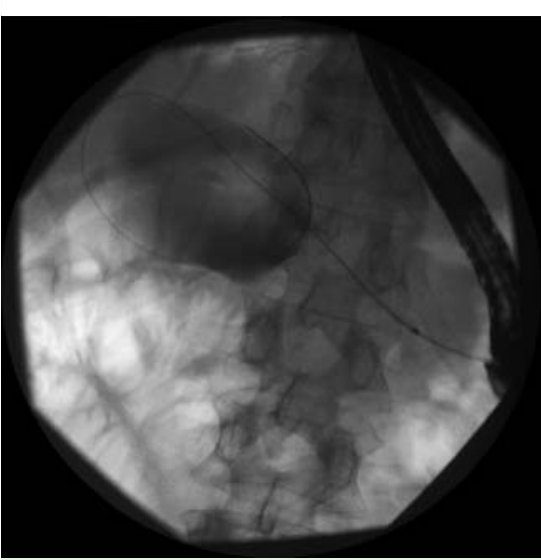

Fig. 3 Guide wire inside the pseudocyst. 


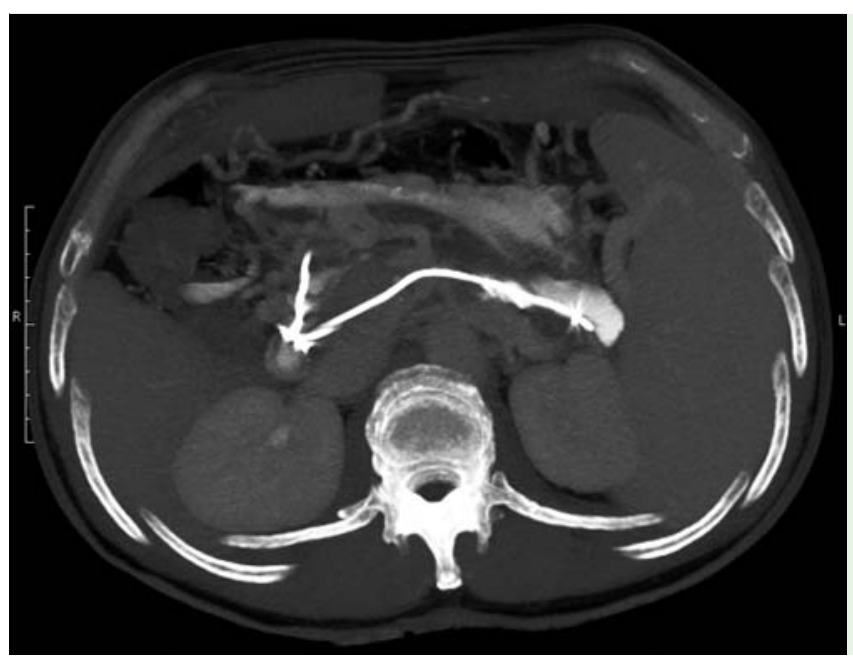

Fig. 5 Computed tomography (CT) scan after transpapillary transpancreatic drainage.
Endoscopy_UCTN_Code_TTT_1AR_2AI

Competing interests: None

\section{Kraft ${ }^{1}$, P. Simon' ${ }^{1}$, C. Nitsche ${ }^{1}$, S. Runge ${ }^{1}$, N. Hahn' ${ }^{1}$, R. Puls ${ }^{2}$, M. M. Lerch ${ }^{1}$}

${ }^{1}$ Department of Internal Medicine A, University Medicine Greifswald, Greifswald, Germany

${ }^{2}$ Department of Radiology and Neuroradiology, University Medicine Greifswald, Greifswald, Germany

Bibliography

DOI http://dx.doi.org/

10.1055/s-0032-1306793

Endoscopy 2012; 44: E186-E187

(C) Georg Thieme Verlag KG

Stuttgart · New York

ISSN 0013-726X

Corresponding author

M. Kraft

Department of Internal Medicine A

University Medicine Greifswald

Ernst-Moritz-Arndt Universität Greifswald

Friedrich-Löffler-Str. 23a

17475 Greifswald

Germany

Fax: +49-3834-867234

matthias.kraft@uni-greifswald.de 